\title{
The Interaction of Oxytocin and Social Support, Loneliness, and Cortisol Level in Major Depression
}

\author{
Tsung-Yu Tsai ${ }^{1, *}$, Huai-Hsuan Tseng ${ }^{1,2, *}$, Mei Hung Chi ${ }^{1}$, Hui Hua Chang ${ }^{3,4,5,6}$, Cheng-Kuan Wu ${ }^{1}$, \\ Yen Kuang Yang ${ }^{1,2,7}$, Po See Chen ${ }^{1,2,8}$
}

Departments of ${ }^{1}$ Psychiatry and ${ }^{5}$ Pharmacy, National Cheng Kung University Hospital, College of Medicine, National Cheng Kung University, ${ }^{2}$ Institute of Behavioral Medicine, ${ }^{3}$ Institute of Clinical Pharmacy and Pharmaceutical Sciences, and ${ }^{4}$ School of Pharmacy, College of Medicine, National Cheng Kung University, Tainan, Departments of ${ }^{6}$ Pharmacy and ${ }^{7}$ Psychiatry, National Cheng Kung University Hospital, Dou-Liou Branch, Yunlin, ${ }^{8}$ Addiction Research Center, National Cheng Kung University, Tainan, Taiwan

\begin{abstract}
Objective: Loneliness is a specific risk factor for depressive symptoms and suicidal behavior. The present study examined whether the serum oxytocin level would interact with social support and buffers loneliness and hypothalamic-pituitary-adrenal (HPA)-axis activity in drug-naïve patients with major depressive disorder (MDD).

Methods: Twenty-six patients with MDD (male:female = 3:23; mean age, $45.54 \pm 12.97$ years) were recruited. The 17-item Hamilton Depression Rating Scale, UCLA Loneliness Scale and self-reported Measurement of Support Function Questionnaire were administered. Serum oxytocin and cortisol levels were assessed using a commercial immunoassay kits.

Results: In MDD patients, a negative association was found between degrees of social support and loneliness ( $\beta=$ $-0.39, p=0.04)$. The interaction between social support and serum oxytocin level was negatively associated with loneliness $(\beta=-0.50, p=0.017)$ and serum cortisol level $(\beta=-0.55, p=0.020)$ after adjusting for age. Follow-up analyses showed that the association between higher social support and lower loneliness was observed only in the higher-oxytocin group $(r=-0.75, p=0.003)$ but not in the lower group $(r=-0.19, p=0.53)$. The significance remained after further adjusting for sex and depression severity.

Conclusion: Low oxytocin level is a vulnerability factor for the buffering effect of social support for loneliness and aberrant HPA-axis activity in MDD patients.
\end{abstract}

KEY WORDS: Cortisol; Loneliness; Major depressive disorder; Oxytocin; Social support.

\section{INTRODUCTION}

Loneliness is a crucial determent factor for common mental disorders and depression [1,2]. In major depressive disorder (MDD) patients, their depression severity is associated with degrees of loneliness [3]. Furthermore, loneliness could be an important risk factor for suicide among depression [4]. Loneliness is also associated with poor prognosis in late-life depression [5]. The presence of

Received: December 26, 2018/ Revised: March 11, 2019 Accepted: April 4, 2019

Address for correspondence: Po See Chen Department of Psychiatry, National Cheng Kung University Hospital, 138 Sheng Li Road, North Dist., Tainan 70403, Taiwan E-mail: chenps@mail.ncku.edu.tw

ORCID: https://orcid.org/0000-0003-4963-578X

*These authors contributed equally to this study. loneliness is associated with a flattening of the diurnal cortisol rhythm [6].

The negative association between loneliness and perceived social support is well-documented [7-9]. Higher loneliness or lower perceived social support at baselines are both significant predictors of depression remission [9], while loneliness might be a better predictor of depression than social support [10]. Compared the direct effects of loneliness on depression, the effects of social support is indirect and it plays a mediating role between loneliness and depression $[11,12]$.

Evidence suggests that the influence of perceived social support on health is mediated by the neuropeptide oxytocin $[13,14]$. Oxytocin increases trust, regulates stress, facilitates emotion recognition, increases prosocial behavior, and attenuates loneliness $[2,15]$. Oxytocin modu-

(ㄷ) This is an Open-Access article distributed under the terms of the Creative Commons Attribution Non-Commercial License (http://creativecommons.org/licenses/by-nc/4.0) which permits unrestricted non-commercial use, distribution, and reproduction in any medium, provided the original work is properly cited. 
lates the emotional functions of the amygdala and hypothalamus $[16,17]$. On behavioral level, oxytocin facilitates expression of the levels of sociality, prosocial behavior, and stress regulation thorugh the effects on hypothalamic-pituitary-adrenal (HPA) axis [13,18,19].

Anxiety and depression are part of the adverse consequences of HPA axis dysfunction [20]. Increased morning cortisol [21], cortisol awakening response and average daily cortisol had been reported in depressed individuals [22]. Both higher serum oxytocin level and better social interaction are associated with reduced activity in the HPA axis. Studies also suggest that the alleviating effect of social support is mediated by oxytocin $[14,23,24]$. The negative correlations between oxytocin level and the core symptoms of depression have been reported [25]. Furthermore, oxytocin levels in depressed female were associatied with depression and anxiety symptoms severity [26]. Its enhancing effect on social affiliation may play a crucial role in both pathophysiology and therapeutic effect in MDD [27].

Despite that oxytocin-based social buffering effect of stress is observed in both animal [28] and human studies [13], the effect has not been examined in patients with depression. Assuming that lack of social support could be a risk factor for the poor outcome among patients with MDD, in the current study we aimed to examine the buffer effect of oxytocin, in the link between lack of social support with loneliness, as well as poor HPA-axis activity in MDD patients. Well-established statistical models were employed [29], to test that whether there is significant interaction between the serum oxytocin level and social support on the loneliness and HPA-axis activity in MDD patients.

\section{METHODS}

\section{Ethics Statement}

The research protocol was approved by the Ethical Committee for Human Research at the National Cheng Kung University (B-ER-106-374), and written informed consent was obtained from each subject before any procedures were performed.

\section{Subjects}

In total, 26 drug-naïve MDD patients were recruited from 2013 to 2015. MDD outpatients aged 21 to 63 years who met the Diagnostic and Statistical Manual of Mental Disorders, fourth edition (DSM-IV), diagnostic criteria, underwent a Mini International Neuropsychiatric Interview (MINI), and completed the 17-item Hamilton Depression Rating Scale (HAM-D) were enrolled consecutively by trained psychiatrists. Patients who met the following criteria were excluded: 1) monoamine oxidase inhibitor or any class of antidepressant treatment prior to entering the study; 2) a DSM-IV diagnosis of substance abuse within the past three months; 3) an organic mental disease, mental retardation or dementia; 4 ) a serious surgical condition or physical illness; and 5) patients who were pregnant or breastfeeding.

\section{Plasma Level of Oxytocin and Serum Level of Cortisol}

Fasting blood samples were collected between 08:00 and 10:00 in the morning. Blood samples for plasma oxytocin assay were collected from the antecubital vein into pre-chilled 5-ml ethylenediaminetetraacetic acid tubes with $250 \mathrm{KIU}$ of apoprotinin and refrigerated until processing. Plasma was isolated by centrifugation at $1,800 \times g$ for 15 minutes at $4^{\circ} \mathrm{C}$ and stored in aliquots at $-70^{\circ} \mathrm{C}$. Oxytocin immune reactivity levels were quantified in duplicate using a commercial oxytocin ELISA kit (Enzo Life Sciences, Farmingdale, NY, USA; formerly Assays Design, Ann Arbor, MI, USA). The detection range was from 12.35 to $1,000 \mathrm{pg} / \mathrm{ml}$. The sensitivity, i.e., the minimum detectable dose of oxytocin, of our assay was $4.92 \mathrm{pg} / \mathrm{ml}$. No extraction was conducted. The intra-assay precision and inter-assay precision of the assay were lower than $10 \%$ and $12 \%$, respectively (coefficient of variance $[\mathrm{CV}](\%)=$ standard deviation/mean $\times 100$; intra-assay: CV $<10 \%$; inter-assay: CV $<12 \%$ ).

Fasting blood sample for cortisol was collected between 08:00 and 10:00 in the morning, too. Cortisol level was assessed using a commercial radioimmunoassay kit (sensitivity, $0.2 \mathrm{ng} / \mathrm{dl}$ ) (Immulite Cortisol; DPC ${ }^{\mathbb{R}}$ Biermann, Bad-Nauheim, Germany). The inter- and intra-assay coefficients of variation were $<7.8 \%$ and $<7.7 \%$, respectively.

\section{Measurement of Support Function (MSF)}

The self-reported MSF questionnaire [30] was adopted for use in this study. This measurement has been used previously to assess social support status, and consists of four subscales: perceived crisis support, perceived routine 
support, received crisis support, and received routine support. In this study, the sum score was employed and the Cronbach's alpha of this sample is 0.91. A higher score indicated the receipt of more social support.

\section{The University of California, Los Angeles (UCLA) Loneliness Scale}

UCLA Loneliness Scale (version 3) has previously been used to measure loneliness [31]. This scale is one of the most widely used loneliness measures and has a high reliability and validity. This scale contains 20 items and the Cronbach's alpha of this sample is 0.65 . Participants rated how often they felt the way described in the item using a four-point Likert scale ranging from 'never' to 'often'.

\section{Hamilton Depression Rating Scale (HAM-D)}

The 17-item HAM-D is widely available for measurement of the severity of depressive symptoms. This scale provides comprehensive coverage of depressive symptoms, and has strong psychometric properties, high concurrent and differential validities, and a strong reliability [32]. Participants rated the severity of depressive symptoms using a three- to five-point scale for each item. The total score ranged from 0 to 52 and the Cronbach's alpha of this sample is 0.77 .

\section{Statistics}

In the first multiple linear regression model, we examined the cross-sectional associations of loneliness with social support and serum oxytocin level, adjusting for age (model 1) [33-35]. Building on model 1, we repeated the analysis with an additional term for the effect of inter- action between social support and oxytocin to examine the association between the interaction term and loneliness, adjusting for age (model 2). The interaction term was centered before entering the model. As sex and level of depression may also influence subjective loneliness, in the third analysis (model 3), we tested the interaction of oxytocin and social support by repeating model 2 with further adjustment for sex and HAM-D score. To examine whether the interaction of oxytocin with social support is also associated with the stress-related biological marker, serum cortisol level, we performed a parallel set of regression analyses, including models 1, 2, and 3, with the serum cortisol level as the outcome variable. For linear regression models, we reported unstandardized coefficient estimates (B) and standardized estimates $(\beta)$ with significance test results ( $p$ values).

To increase the interpretability of the oxytocin-social support interaction effect on loneliness and cortisol level, we performed an additional analysis by splitting the subjects into groups of low and high oxytocin levels at the median $(22.5 \mathrm{pg} / \mathrm{ml})$. Pearson's correlations between loneliness, cortisol level and social support were examined in the low and high oxytocin level groups separately. Statistical analyses were conducted using SPSS 17.0 (SPSS Inc., Chicago, IL, USA). Significance was assumed at $p<$ 0.05 .

\section{RESULTS}

The 26 MDD patients were predominantly female (male:female $=3: 23$ ), with a mean age of 45.54 years. The other demographic, clinical, and laboratory data are sum-

Table 1. Demographic data and comparison of low and high oxytocin level groups

\begin{tabular}{lccccc}
\hline \multicolumn{1}{c}{ Variable } & Total $(\mathrm{n}=26)$ & Low $(\mathrm{n}=13)$ & High $(\mathrm{n}=13)$ & \multicolumn{2}{c}{ Statistic } \\
\cline { 4 - 6 } & & & & $\mathrm{t}^{\mathrm{a}} / \chi^{2}$ & $p$ \\
\hline Age $(\mathrm{yr})$ & $45.54 \pm 12.97$ & $45.38 \pm 13.36$ & $45.69 \pm 13.10$ & -0.06 & 0.95 \\
Sex, male/female & $3 / 23$ & $1 / 12$ & $2 / 11$ & 0.38 & 0.54 \\
Oxytocin level $(\mathrm{pg} / \mathrm{ml})$ & $27.98 \pm 17.80$ & $14.20 \pm 6.84$ & $41.76 \pm 14.19$ & -6.31 & $<0.001$ \\
Loneliness & $52.19 \pm 11.23$ & $51.23 \pm 10.49$ & $53.15 \pm 12.27$ & -0.43 & 0.67 \\
MSF sum score & $102.96 \pm 18.64$ & $100.69 \pm 23.94$ & $105.23 \pm 11.82$ & -0.61 & 0.55 \\
HAM-D & $17.46 \pm 6.38$ & $15.85 \pm 5.87$ & $19.08 \pm 6.69$ & -1.31 & 0.20 \\
Cortisol level (mg/dl) & $15.06 \pm 6.31$ & $15.23 \pm 6.74$ & $14.89 \pm 6.13$ & 0.13 & 0.89 \\
\hline
\end{tabular}

Values are presented as mean \pm standard deviation or number only.

Patients were split into groups with low and high levels of oxytocin according to the median $(22.5 \mathrm{pg} / \mathrm{ml})$.

MSF, Measurement of Support Function; HAM-D, Hamilton Depression Rating Scale.

${ }^{a}$ The results were similar when the Mann-Whitney $U$ test was used. 
marized in Table 1.

\section{Effect of Social Support and Oxytocin Interaction on Subjective Loneliness}

In model 1, we assessed the associations between social support, oxytocin level, and loneliness, taking age into consideration. We found that a younger age and lower social support, but not oxytocin level, were associated with a greater UCLA Loneliness Scale score (Table 2). After adding the interaction term in model 2, we observed that effect of interaction between social support and serum oxytocin level was negatively associated with UCLA loneliness score $(\beta=-0.50, p=0.017)$, in addition to social support alone ( $\beta=-0.66, p=0.002)$. The negative association between the social support-oxytocin interaction and loneliness remained significant $(\beta=-0.50, p=$ $0.024)$ after further adjustment for $\operatorname{sex}(\beta=0.10, p=0.56)$ and HAM-D score $(\beta=0.12, p=0.50)$ in model 3 .

In an additional analysis, we split the MDD subjects into groups of low and high oxytocin levels. There was no significant difference in the UCLA loneliness score between the two groups ( $t=-0.43, p=0.67$ ) (Table 1). A significant negative correlation between social support and loneliness was observed only in the group with a high oxytocin level $(r=-0.75, p=0.003)$, and not in the low oxytocin level group $(r=-0.19, p=0.53)$ (Table 3, Fig. 1$)$.

\section{Effect of Social Support and Oxytocin Level Interaction on Serum Cortisol Level}

Based on our finding in this study of a negative correlation between the interaction of oxytocin with social support and loneliness, we further explored the correlation with the interaction of oxytocin level and social support by repeating the analyses of the biological stress marker of serum cortisol level (Table 2). We did not observe a significant association between social support or oxytocin level and serum cortisol level after controlling for age (model 1); however, after considering the interaction terms, we observed a negative relationship between the interaction of oxytocin and social support and cortisol level in both model $2(\beta=-0.55, p=0.020)$ and model 3 $(\beta=-0.52, p=0.026)$, in addition to a negative relation-

Table 3. Pearson correlations of the MSF score with loneliness and cortisol level in low and high oxytocin levels groups

\begin{tabular}{ccccccc}
\hline \multirow{2}{*}{ Association } & \multicolumn{2}{c}{$\begin{array}{c}\text { Low oxytocin } \\
\operatorname{group}(\mathrm{n}=13)\end{array}$} & & \multicolumn{2}{c}{$\begin{array}{c}\text { High oxytocin } \\
\operatorname{group}(\mathrm{n}=13)\end{array}$} \\
\cline { 2 - 3 } \cline { 5 - 6 } & \multicolumn{2}{c}{$r^{\mathrm{a}}$} & $p$ & & $r^{\mathrm{a}}$ & $p$ \\
\hline $\begin{array}{c}\text { Between MSF and } \\
\text { loneliness } \\
\begin{array}{c}\text { Between MSF and } \\
\text { cortisol }\end{array}\end{array}$ & -0.19 & 0.53 & & -0.75 & 0.003 \\
\hline
\end{tabular}

MSF, Measurement of Support Function.

'The results were similar when Spearman's rho correlation or partial correlation, controlling age, sex, and Hamilton Depression Rating Scale was used.

Table 2. Three multiple linear regression models of cortisol level and loneliness

\begin{tabular}{|c|c|c|c|c|c|c|c|c|c|}
\hline & \multicolumn{3}{|c|}{ Model 1} & \multicolumn{3}{|c|}{ Model 2} & \multicolumn{3}{|c|}{ Model 3} \\
\hline & B & $\beta$ & $p$ & B & $\beta$ & $p$ & B & $\beta$ & $p$ \\
\hline & \multicolumn{9}{|c|}{ Dependent variable: Loneliness } \\
\hline Age & -0.39 & -0.45 & $0.021^{*}$ & -0.35 & -0.40 & $0.023^{*}$ & -0.31 & -0.35 & 0.06 \\
\hline MSF & -0.23 & -0.39 & $0.040^{*}$ & -0.40 & -0.66 & $0.002 *$ & -0.39 & -0.65 & $0.004 *$ \\
\hline Oxytocin & 0.10 & 0.16 & 0.38 & 0.07 & 0.11 & 0.51 & 0.03 & 0.04 & 0.83 \\
\hline MSF $\times$ oxytocin & & & & -0.02 & -0.50 & $0.017^{*}$ & -0.02 & -0.50 & $0.024 *$ \\
\hline Sex & & & & & & & 0.04 & 0.10 & 0.56 \\
\hline \multirow[t]{2}{*}{ HAM-D } & & & & & & & 0.22 & 0.12 & 0.50 \\
\hline & \multicolumn{9}{|c|}{ Dependent variable: Cortisol level } \\
\hline Age & -0.13 & -0.27 & 0.20 & -0.10 & -0.21 & 0.26 & -0.06 & -0.12 & 0.53 \\
\hline MSF & -0.07 & -0.22 & 0.29 & -0.18 & -0.52 & $0.025^{*}$ & -0.16 & -0.47 & $0.038^{*}$ \\
\hline Oxytocin & 0.05 & 0.15 & 0.46 & 0.03 & 0.10 & 0.61 & -0.03 & -0.08 & 0.71 \\
\hline MSF $\times$ oxytocin & & & & -0.01 & -0.55 & $0.020^{*}$ & -0.01 & -0.52 & $0.026^{*}$ \\
\hline Sex & & & & & & & 0.03 & 0.16 & 0.39 \\
\hline HAM-D & & & & & & & 0.35 & 0.36 & 0.08 \\
\hline
\end{tabular}

Unstandardized coefficient estimates (B) and standardized estimates ( $\beta$ ) were both assessed.

MSF, Measurement of Support Function; HAM-D, Hamilton Depression Rating Scale.

$* p<0.05$. 


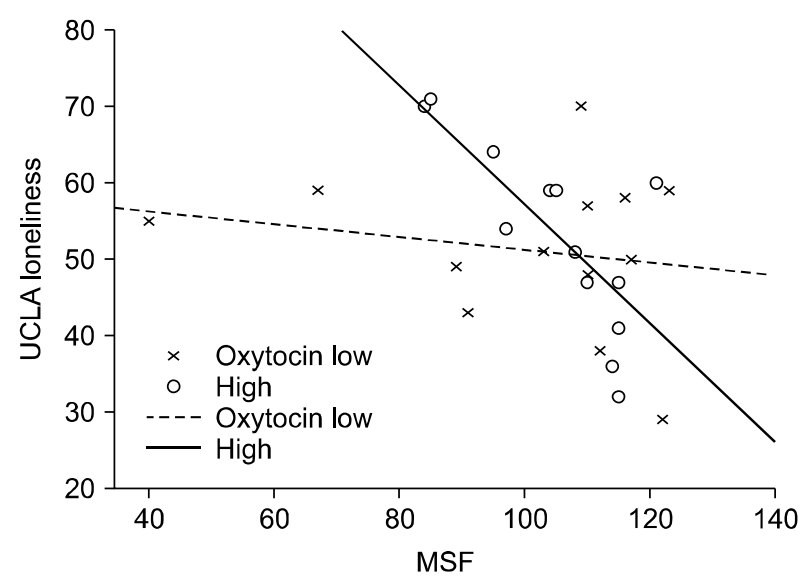

Fig. 1. Scatter plot between Measurement of Support Function (MSF) score and the University of California, Los Angeles (UCLA) loneliness score in both of high and low oxytocin groups.

ship with social support alone $(\beta=-0.52, p=0.025$ in model $2 ; \beta=-0.47, p=0.038$, respectively).

In the additional analysis in which subjects were split into two groups according to oxytocin level, there was no significant difference in the serum cortisol level between the patients with a high oxytocin level and those with a low level $(t=0.13, p=0.89)$ (Table 1). We did not observe a significant association between social support and serum cortisol level in either the low or high oxytocin level group $(r=-0.11, p=0.73$ and $r=-0.41, p=0.16$, respectively) (Table 3).

\section{DISCUSSION}

In a female-predominant sample of patients with MDD, we observed an interaction effect between serum oxytocin level and social support, which was significantly associated with lower subjective loneliness. Follow-up analyses further revealed a negative association between social support and loneliness which only observed in the high oxytocin level subgroup. Similarly, social supportoxytocin interaction was also negatively associated with serum cortisol level, a biological marker of stress.

Our first regression model (model 1) replicated previous observations that greater social support is associated with lower loneliness in a healthy population [36] and in elder depressive patients [11]. Prior research has observed the association between greater loneliness and poorer social support and depression; however, limited studies have investigated the concurrent association be- tween social support and loneliness in adult depressive patients [37]. In line with our hypothesis, we found a negative association between social support and loneliness in model 2 and model 3, and our results further suggested that oxytocin might facilitate the effect of social support in terms of buffering loneliness, even after adjusting for age (model 2), sex, and level of depression (model 3). Our results echoed findings of an oxytocin-based social buffering of stress cues in animal [28] and human studies [13]. The results suggested that social support may alleviate loneliness only in the existence of adequate oxytocin level in depressive patients.

As hypothesized, we observed a negative correlation between the social support-oxytocin level interaction and the serum cortisol level in model 2 and model 3, similar to that seen with loneliness. The negative correlation between social support and cortisol level was stronger in the high oxytocin level group $(r=-0.41)$ than in the low oxytocin level group $(r=-0.11)$, supporting the hypothesis of an oxytocin-based social buffering effect on stress regulation in depressive patients. This result suggests an oxytocin-based buffering effect to the current stress level, as to stress responsiveness observed by Heinrichs et al. [24], which employed intranasal oxytocin administration in a healthy male population.

Interestingly, we did not find a significant correlation between oxytocin and loneliness in MDD patients. The negative correlation with loneliness exists at the social support-oxytocin interaction level rather than the main effect of oxytocin may reflect a context-related prosocial effect of oxytocin. The interaction of oxytocin and social support had more prominent effects to suppress the stress-induced cortisol response and anxiety than social support or oxytocin alone [24]. In contrast, a negative or null effect was observed while the administration of oxytocin was in a negative or non-social context [38-40]. In sum, the effects of oxytocin are social environment dependent [13] and vary in different level of social support.

The exact role of oxytocin in MDD is a complex question. Depressed female is more likely to display a dysregulated pattern of peripheral oxytocin release than controls [26], and has a reduced plasma oxytocin level $[41,42]$. Furthermore, a negative correlation between oxytocin and the severity of depressive symptoms was identified [25]. In summary, the level of oxytocin seems to be lower in individuals with more severe depressive 
symptoms, particularly in females. Given that depression severity is associated with degrees of loneliness [3], we expected that depression severity may moderate the association between the social support, oxytocin level and loneliness. However, in our sample, the severity of depression did not affect the alleviating effect of social support and its interaction effect with oxytocin on loneliness. Similarly, our results suggest that the synergistic effect of social support and oxytocin level on the HPA axis may be relatively independent to the severity of depression. These results imply a similar buffering effect of social support on loneliness and HPA axis activities in MDD patients and in healthy populations $[6,11,43]$.

There were some limitations of the current study. First, the correlational analyses we applied limited the extent to which causal inferences may be made. Second, the sample size was limited, and the results may not be able to be generalized to other populations. Third, much of the variability in loneliness owing to objective social relationships, personality traits, or environmental interactions was not accounted for.

We replicated the buffering effect of social support on loneliness and HPA axis activity, and identified a facilitating factor, higher serum oxytocin level, that had synergistic effect with social support to alleviate the loneliness in drug-naïve female patients with depression. Those depressive patients with lower level of oxytocin and socially unsupported were likely to experience higher level of loneliness and HPA axis hyperactivity. These findings will inform a new direction of study into the neurobiology of oxytocin that may significantly influence the effect of social support, and may indicate a more effective route for bio-psycho-social intervention and prevention in major depression.

\section{- Acknowledgments}

This work was supported by the Ministry of Science and Technology, Taiwan (MOST 103-2320-B-006-013, MOST 104-2321-B-006-031, MOST 105-2321-B-006-020, MOST 106-2320-B-006-052, and MOST 107-2320-B-006-016) and National Cheng Kung University Hospital (NCKUH10703005). The authors wish to thank Dr. Shih-Hsien Lin for his advice and Mr. Chien Ting Lin for his administrative support.

\section{Conflicts of Interest}

No potential conflict of interest relevant to this article was reported.

\section{Author Contributions}

Po See Chen designed the study and wrote the protocol. Yen Kuang Yang helped to design the protocol. Huai-Hsuan Tseng and Hui Hua Chang contributed to the statistical analyses. Tsung-Yu Tsai wrote the first draft of the manuscript. Mei Hung Chi, Cheng-Kuan Wu, Yen Kuang Yang, and Po See Chen managed the data collection. All authors interpreted the results and helped to revise the manuscript.

\section{ORCID}

Tsung-Yu Tsai https://orcid.org/0000-0003-4593-2904

Huai-Hsuan Tseng https://orcid.org/0000-0002-5213-1585 Mei Hung Chi https://orcid.org/0000-0001-5408-5466 Hui Hua Chang https://orcid.org/0000-0001-7866-5481 Cheng-Kuan Wu https://orcid.org/0000-0002-6740-956X Yen Kuang Yang https://orcid.org/0000-0001-9355-9636 Po See Chen https://orcid.org/0000-0003-4963-578X

\section{REFERENCES}

1. Cacioppo JT, Hughes ME, Waite LJ, Hawkley LC, Thisted RA. Loneliness as a specific risk factor for depressive symptoms: cross-sectional and longitudinal analyses. Psychol Aging 2006:21:140-151.

2. Cacioppo S, Grippo AJ, London S, Goossens L, Cacioppo JT. Loneliness: clinical import and interventions. Perspect Psychol Sci 2015;10:238-249.

3. Singh A, Misra N. Loneliness, depression and sociability in old age. Ind Psychiatry J 2009;18:51-55.

4. Stickley A, Koyanagi A. Loneliness, common mental disorders and suicidal behavior: findings from a general population survey. J Affect Disord 2016;197:81-87.

5. Holvast $\mathrm{F}$, Burger $\mathrm{H}$, de Waal MM, van Marwijk HW, Comijs $\mathrm{HC}$, Verhaak PF. Loneliness is associated with poor prognosis in late-life depression: longitudinal analysis of the Netherlands study of depression in older persons. J Affect Disord 2015;185:1-7.

6. Doane LD, Adam EK. Loneliness and cortisol: momentary, day-to-day, and trait associations. Psychoneuroendocrinology 2010;35:430-441.

7. Rook KS. Research on social support, loneliness, and social isolation: toward an integration. Rev Personal Soc Psychol 1984;5:239-264.

8. Salimi A, Bozorgpour F. Perceived social support and so- 
cial-emotional loneliness. Procedia Soc Behav Sci 2012;69. 2009-2013.

9. Wang J, Mann F, Lloyd-Evans B, Ma R, Johnson S. Associations between loneliness and perceived social support and outcomes of mental health problems: a systematic review. BMC Psychiatry 2018;18:156.

10. Alpass FM, Neville S. Loneliness, health and depression in older males. Aging Ment Health 2003;7:212-216.

11. Liu L, Gou Z, Zuo J. Social support mediates loneliness and depression in elderly people. J Health Psychol 2016;21:750758.

12. Hagerty BM, Williams RA. The effects of sense of belonging, social support, conflict, and loneliness on depression. Nurs Res 1999:48:215-219.

13. Olff M, Frijling JL, Kubzansky LD, Bradley B, Ellenbogen MA, Cardoso $\mathrm{C}$, et al. The role of oxytocin in social bonding, stress regulation and mental health: an update on the moderating effects of context and interindividual differences. Psychoneuroendocrinology 2013;38:1883-1894.

14. Kubzansky LD, Mendes WB, Appleton A, Block J, Adler GK. Protocol for an experimental investigation of the roles of oxytocin and social support in neuroendocrine, cardiovascular, and subjective responses to stress across age and gender. BMC Public Health 2009;9:481.

15. Kosfeld M, Heinrichs M, Zak PJ, Fischbacher U, Fehr E. Oxytocin increases trust in humans. Nature 2005:435:673676

16. Cochran DM, Fallon D, Hill M, Frazier JA. The role of oxytocin in psychiatric disorders: a review of biological and therapeutic research findings. Harv Rev Psychiatry 2013;21:219247.

17. Stoop R. Neuromodulation by oxytocin and vasopressin. Neuron 2012;76:142-159.

18. Carter CS. Oxytocin pathways and the evolution of human behavior. Annu Rev Psychol 2014;65:17-39.

19. Sivukhina EV, Jirikowski GF. Magnocellular hypothalamic system and its interaction with the hypothalamo-pituitary-adrenal axis. Steroids 2016;111:21-28.

20. Doane LD, Mineka S, Zinbarg RE, Craske M, Griffith JW, Adam EK. Are flatter diurnal cortisol rhythms associated with major depression and anxiety disorders in late adolescence? The role of life stress and daily negative emotion. Dev Psychopathol 2013;25:629-642.

21. Bhagwagar Z, Hafizi S, Cowen PJ. Increased salivary cortisol after waking in depression. Psychopharmacology (Berl) 2005; 182:54-57.

22. Dienes KA, Hazel NA, Hammen CL. Cortisol secretion in depressed, and at-risk adults. Psychoneuroendocrinology 2013; 38:927-940.

23. Carter CS. Neuroendocrine perspectives on social attachment and love. Psychoneuroendocrinology 1998;23:779-818.

24. Heinrichs M, Baumgartner T, Kirschbaum C, Ehlert U. Social support and oxytocin interact to suppress cortisol and sub- jective responses to psychosocial stress. Biol Psychiatry 2003; 54:1389-1398.

25. Scantamburlo G, Hansenne M, Fuchs S, Pitchot W, Maréchal $\mathrm{P}$, Pequeux C, et al. Plasma oxytocin levels and anxiety in patients with major depression. Psychoneuroendocrinology 2007;32:407-410.

26. Cyranowski JM, Hofkens TL, Frank E, Seltman H, Cai HM, Amico JA. Evidence of dysregulated peripheral oxytocin release among depressed women. Psychosom Med 2008;70: 967-975.

27. Arletti R, Bertolini A. Oxytocin acts as an antidepressant in two animal models of depression. Life Sci 1987;41:17251730.

28. Smith AS, Wang Z. Hypothalamic oxytocin mediates social buffering of the stress response. Biol Psychiatry 2014;76:281288.

29. Cohen S, Wills TA. Stress, social support, and the buffering hypothesis. Psychol Bull 1985;98:310-357.

30. Lin N, Ye X, Ensel WM. Social support and depressed mood: a structural analysis. J Health Soc Behav 1999;40:344-359.

31. Russell DW. UCLA Loneliness Scale (version 3): reliability, validity, and factor structure. J Pers Assess 1996;66:20-40.

32. Gonzalez JS, Shreck E, Batchelder A. Hamilton Rating Scale for Depression (HAM-D). In: Gellman MD, Turner JR, editors. Encyclopedia of behavioral medicine. New York:Springer;2013. p.887-888.

33. Segrin C. Age moderates the relationship between social support and psychosocial problems. Hum Commun Res 2003; 29:317-342.

34. Pinquart M, Sörensen S. Risk factors for loneliness in adulthood and old age: a meta-analysis. In: Shohov SP, editor. Advances in psychology research. Vol. 19. Hauppauge, NY:Nova Science Publishers;2003. p.111-143.

35. Yang K, Victor C. Age and loneliness in 25 European nations. Ageing Soc 2011;31:1368-1388.

36. Segrin C, Passalacqua SA. Functions of loneliness, social support, health behaviors, and stress in association with poor health. Health Commun 2010;25:312-322.

37. van den Brink RHS, Schutter N, Hanssen DJC, Elzinga BM, Rabeling-Keus IM, Stek ML, et al. Prognostic significance of social network, social support and loneliness for course of major depressive disorder in adulthood and old age. Epidemiol Psychiatr Sci 2018;27:266-277.

38. Cardoso C, Valkanas H, Serravalle L, Ellenbogen MA. Oxytocin and social context moderate social support seeking in women during negative memory recall. Psychoneuroendocrinology 2016;70:63-69.

39. Scheele D, Kendrick KM, Khouri C, Kretzer E, Schläpfer TE, Stoffel-Wagner B, et al. An oxytocin-induced facilitation of neural and emotional responses to social touch correlates inversely with autism traits. Neuropsychopharmacology 2014; 39:2078-2085.

40. Bartz J, Simeon D, Hamilton H, Kim S, Crystal S, Braun A, et 
al. Oxytocin can hinder trust and cooperation in borderline personality disorder. Soc Cogn Affect Neurosci 2011;6:556563.

41. van Londen L, Goekoop JG, van Kempen GM, FrankhuijzenSierevogel AC, Wiegant VM, van der Velde EA, et al. Plasma levels of arginine vasopressin elevated in patients with major depression. Neuropsychopharmacology 1997;17:284-292.

42. Slattery DA, Neumann ID. Oxytocin and major depressive disorder: experimental and clinical evidence for links to aetiology and possible treatment. Pharmaceuticals (Basel) 2010; 3:702-724.

43. Guan SA, Bower JE, Almeida DM, Cole SW, Dahl RE, Irwin $\mathrm{MR}$, et al. Parental support buffers the association of depressive symptoms with cortisol and C-reactive protein during adolescence. Brain Behav Immun 2016;57:134-143. 